\title{
La reforma energética en México y su impacto en la ocupación superficial para el aprovechamiento de los recursos energéticos
}

The energy reform in Mexico and its impact on land occupation for the utilisation of energy resources

Autores: Rosa María Díaz López, Verónica Rivera de la Rosa DOI: https://doi.org/10.25058/1794600X.1917 


\title{
LA REFORMA ENERGÉTICA EN MÉXICO Y SU IMPACTO EN LA OCUPACIÓN SUPERFICIAL PARA EL APROVECHAMIENTO DE LOS RECURSOS ENERGÉTICOS*
}

\author{
The energy reform in Mexico and its impact on land occupation \\ for the utilisation of energy resources
}

A reforma energética no México e seu impacto na ocupação da superfície para o uso de recursos energéticos

\author{
Rosa María Díaz López ${ }^{a}$ \\ rosy4343@hotmail.com \\ Verónica Rivera de la Rosab \\ veronica.riveradl@gmail.com
}

Fecha de recepción: 2 de noviembre de 2018 Fecha de revisión: 16 de agosto de 2019 Fecha de aceptación: 16 de noviembre de 2020

\section{RESUMEN}

La realización de las actividades del sector energético, específicamente en materia de exploración y extracción de hidrocarburos, deberán ejecutarse bajo los criterios de equidad social, productividad y sustentabilidad, lo cual requiere la existencia de un marco constitucional sólido que regularice el uso y la ocupación superficial para la ejecución de proyectos del sector energético, toda vez que existen principios que se deben considerar, entre los que destacan: el uso superficial es temporal en algunos proyectos; las comunidades, a menudo, se ven afectadas por un período de tiempo más prolongado que el estimado requerido para llevar a cabo la actividad real de uso de suelo; durante las actividades de uso de suelo, se deben conservar los recursos naturales afectados; la tierra debe ser restaurada a su condición original, o condición alternativa adecuada, después de que se haya concluido la actividad de ocupación superficial; y cualquier actividad de uso de suelo tiene el potencial de afectar el medio ambiente, positiva o negativamente. Hay que hacer una descripción

\footnotetext{
* Artículo de reflexión.

a. Licenciada en Derecho por la Universidad Nacional Autónoma de México (UNAM), Maestra en Derecho (UNAM), Doctora en Derecho (UNAM). Investigador en el Sistema Nacional de Investigadores (SNI-Nivel 1), Investigador Titular en el Centro de Investigación de Tecnología Jurídica y Criminológica (CITEJYC) de la Universidad Autónoma de Nuevo León (UANL), Catedrática en el área de Posgrado de la Facultad de Derecho y Criminología de la UANL. Profesora visitante en la Université de Lille en Francia. Autora del libro El Ombudsman de la salud en México (Instituto de Investigaciones Jurídicas de la UNAM); coautora y coordinadora de la obra: Regulación de servicios públicos y energía (Editorial Porrúa, Asociación Iberoamericana de Estudios de Regulación (ASIER) y la Universidad Autónoma de Nuevo León); coautora y coordinadora del libro: Panorama actual del derecho fiscal y energético en México (Editorial Nueva Jurídica de Colombia y el Colegio Nacional de Profesores e Investigadores de Derecho Fiscal y Finanzas Públicas, A.C.).
}

b. Licenciada en Ciencias del Lenguaje con Acentuación en Traducción e Interpretación por la Facultad de Filosofía y Letras de la Universidad Autónoma de Nuevo León. Colaboradora en proyectos de investigación académica en el Centro de Investigación de Tecnología Jurídica y Criminológica de la Facultad de Derecho y Criminología de la UANL. 
de los posibles impactos ambientales adversos desde la planificación hasta la finalización de la actividad. A cinco años de la implementación de la reforma energética, es imperativo evaluar los alcances y las deficiencias presentadas en los temas de ocupación superficial e impacto social, tras la ejecución de proyectos en las áreas contractuales específicas.

\section{PALABRAS CLAVE}

Reforma energética; Ley de Hidrocarburos; exploración; extracción; recursos energéticos; ocupación superficial; impacto social; sustentabilidad.

\section{ABSTRACT}

The activities of the energy sector, specifically in the exploration and extraction of hydrocarbons must be performed under the criteria of social equity, productivity and sutainability, which requires a solid constitutional framework to regulate the land occupation use for the execution of projects in the energy sector, since there are principles that must be considered, among which the following stand out: the use of land is temporary in some projects; communities oftenly are affected by a period of time longer than the estimated time required to carry out the actual use of the land; during the land use activities the affected natural resources must be preserved, land must be restored to its original condition or to an adequate alternative condition after the activity of land occupation has concluded and any land use activity has the potential to affect the environment, either positively or negatively. A description of the possible adverse environmental impacts from planning to the completion of the activity must be given. After five years of the implementation of the energy reform, it is essential to evaluate the scope and deficiencies regarding land occupation and social impact issues, subsequent to the execution of projects in the specific contractual areas.

\section{KEYWORDS}

Hydrocarbons Law, exploration, extraction, energy resources, land occupation, social impact, sustainability.

\section{RESUMO}

O desempenho das atividades no setor da energia, nomeadamente no domínio da exploração e extração de hidrocarbonetos, deve ser efetuado sob os critérios de equidade social, produtividade e sustentabilidade, o que pressupõe a existência de um sólido quadro constitucional que regule o uso e ocupação. da superfície .para a execução de projectos no sector da energia, uma vez que existem princípios que devem ser tidos em consideração, entre os quais se destacam: o uso da superfície é temporário em alguns projectos; As comunidades são freqüentemente afetadas por um período de tempo mais longo do que a estimativa necessária para realizar a atividade real de uso da terra; Durante as atividades de uso da terra, os recursos naturais afetados devem ser conservados; o terreno deve ser restaurado à sua condição original, ou condição alternativa adequada, após a conclusão da atividade de ocupação superficial; e qualquer atividade de uso da terra tem o potencial de afetar o meio ambiente, positiva ou negativamente. A descrição dos possíveis impactos ambientais adversos deve ser feita desde o planejamento até a conclusão da atividade. Cinco anos após a implantação da reforma energética, é imprescindível avaliar o escopo e as deficiências apresentadas nas áreas de ocupação superficial e impacto social, após a execução dos projetos nas áreas contratuais específicas.

\section{PALAVRAS CHAVE}

Reforma energetica; Lei de Hidrocarbonetos; exploração; Extração; recursos energéticos; ocupação superficial; Impacto social; sustentabilidade.

\section{INTRODUCCIÓN}

Derivado de la reforma energética, se ha presentado un cambio importante para el uso y ocupación superficial de las tierras de los propietarios y poseedores que podrían ser susceptibles para la realización de las actividades en la obtención de energéticos (cabe señalar que permanece sin cambios el principio de utilidad pública de un área estratégica ejercida por el Estado con la excepción de que estas actividades, ya no solo podrán ser llevadas a cabo mediante las industrias del Estado, sino en unión o individualmente 
a través de empresas particulares); la Ley de Hidrocarburos, tiene un apartado especial sobre el uso y ocupación superficial donde se señalan las contraprestaciones, términos, condiciones para el uso, goce o afectación de los terrenos, bienes y derechos, necesarios para la realización de las actividades de exploración, extracción y producción de los energéticos, con un cambio significativo que es la apertura a la negociación y acuerdos, tan cierta es esta posibilidad a negociar, que es evidente deducir que proviene del derecho de los gobernados a buscar un método alterno a la negociación de solución a conflictos (MASC) con fundamento en los artículos $17 \mathrm{y}$ 18 de la Constitución Política de los Estados Unidos Mexicanos (Díaz, 2014, p. 52), entre los propietarios o titulares de terrenos sobre sus bienes o derechos reales (poder jurídico, que tiene por objeto un bien, hay una relación como dijo Baudry Lacantinerie, directa e inmediata entre el titular y la cosa) (En Rojina, 1994, p. 21) y ejidales o comunales (siendo estos últimos una sociedad de interés social integrada por campesinos mexicanos por nacimiento con patrimonio social constituido por tierras); con los ahora recientemente agregados en la reforma energética que son los contratistas (personas morales que pueden participar solas o en unión con la Empresa Productiva del Estado - EPE) y los asignatarios (anteriormente llamadas paraestatales), ahora llamadas Empresas Productivas del Estado (EPE), Petróleos Mexicanos o Comisión Federal de Electricidad.

Es oportuno decir que el presente trabajo es exploratorio y descriptivo de la legislación energética vigente, en materia de ocupación superficial para el aprovechamiento de los recursos energéticos en relación con los hidrocarburos.

El contiene los siguientes apartados: en el primero de ellos, se abordarán aspectos generales del marco institucional y constitucional en el tema de uso y ocupación superficial en materia de hidrocarburos. Más adelante, se hace una exposición de la Ley de Hidrocarburos, la cual establece las condiciones en las que se regula el uso y la ocupación superficial de los terrenos susceptibles para la Exploración, Extracción y Producción de la Industria Petrolera. El artículo 101, fracción IV, de la Ley de Hidrocarburos señala los modelos jurídicos mediante los cuales se realizaran los acuerdos para el uso y ocupación superficial ${ }^{1}$.

En el segundo apartado, se plantea un diagnóstico de las posibles repercusiones económicas, sociales y administrativas que derivan del ejercicio de imponer el interés público sobre la propiedad para el aprovechamiento de los recursos naturales. Se mencionan las repercusiones negativas y positivas al realizar las actividades para la exploración, extracción y producción de los hidrocarburos en las zonas de las comunidades habitadas.

En el tercer apartado, se menciona la importancia que hoy en día tiene la concientización de realizar las actividades con respeto y legalidad en un equilibrio de igualdad de importancia, entre la sociedad, el desarrollo económico y el medio ambiente, por ello se enfatiza en la importancia del impacto social y de la sustentabilidad.

\section{ASPECTOS GENERALES DEL MARCO INSTITUCIONAL Y CONSTITUCIONAL EN EL TEMA DE OCUPACIÓN SUPERFICIAL E HIDROCARBUROS}

En esta sección, se revisarán algunos puntos importantes de las figuras jurídicas relacionadas con la ocupación superficial en proyectos energéticos. En una primera instancia, la definición legal del concepto de propiedad de un bien inmueble, así como de las características facultativas de la Nación de ejercer la propiedad original de la tierra para el aprovechamiento de los elementos naturales (Constitución Federal, 1917, art. 27, párr. 3).

Para comprender el derecho de imponer las modalidades que dicte el interés público es preciso describir éste como: "el conjunto de pretensiones relacionadas con las necesidades colectivas de los miembros de la comunidad y protegidas mediante la intervención directa y permanente del Estado" (Diccionario jurídico mexicano, 1996, p. 1779), sobre el dominio o propiedad de las tierras en México por parte de los particulares, es indispensable señalar la naturaleza jurídica

1. La forma o modalidad de uso, goce, afectación, en su caso, adquisición que se pacte deberá ser idónea para el desarrollo del proyecto en cuestión, según sus características. Al efecto, podrán emplearse las figuras de arrendamiento, servidumbre voluntaria ocupación superficial, ocupación temporal, compraventa, permuta y cualquier otra que no contravenga la ley, artículo 101, fracc. $V$. de la Ley de Hidrocarburos. 
de donde procede la propiedad de la tierra y que tiene origen en el artículo 27 de la Constitucional Política de los Estados Unidos Mexicanos, en su primer párrafo nos señala que la propiedad de las tierras y aguas comprendidas dentro de los límites del territorio nacional, corresponde originariamente a la Nación, la cual ha tenido y tiene el derecho de transmitir el dominio de ellas a los particulares, constituyendo así la propiedad privada.

Bajo este esquema de legitimación analizamos a la propiedad pública, privada y social de la tierra como el resultado que deriva del derecho de la Nación y quien la adquiere se encuentra sujeto en todo momento a la imposición que señale la Nación en las distintas modalidades como ejecutor de los intereses públicos. Embestido así, el Estado con la facultad que le confiere el Artículo 27 de la Constitución (Constitución Federal, Art. 27, párr. III), de ser el que aproveche los elementos naturales con la capacidad de afectar a la propiedad privada, haciendo uso de su derecho original y velar por el interés público (p. 6) de los gobernados con el que podrá limitar el derecho del particular sobre la tierra, para tener acceso a la exploración, explotación y producción para el caso específico de los hidrocarburos.

Ahora bien, después del concepto de propiedad de la tierra en México, pasamos a la definición de bien inmueble que se encuentra descrito en el Código Civil Federal, no sin antes señalar que el concepto de tierra tiene algunos sinónimos utilizados en la legislación mexicana que son: terreno, suelo ${ }^{2}$, aclarado el punto anterior se invoca al artículo 750 del Código Civil del Distrito Federal que señala:

II. suelo y las construcciones adheridas a él; III. Las plantas y árboles, mientras estuvieren unidos a la tierra, y los frutos pendientes de los mismos árboles y plantas mientras no sean separados de ellos por cosechas o cortes regulares;

IV. Todo lo que esté unido a un inmueble de una manera fija, de modo que no pueda separarse sin deterioro del mismo inmueble o del objeto a él adherido;

$\mathrm{V}$. Las estatuas, relieves, pinturas u otros objetos de ornamentación, colocados en

2. Tanto como suelo, terreno y tierra son utilizados en nuestras legislaciones para describir y hacer referencia a la porción de propiedad de un bien inmueble. edificios o heredados por el dueño del inmueble, en tal forma que revele el propósito de unirlos de un modo permanente al fundo.

De lo anterior se desprende que los bienes inmuebles no solo son la proporción de tierra, sino también los bienes muebles que están adheridos y fijados al suelo, por ejemplo: construcciones, plantas, arboles, los frutos de estos, lo que da en conjunto la definición de bien inmueble; que para fines de indemnización se deberá de tomar en cuenta para un avaluó apegado a derecho dentro de un ámbito de legalidad en los términos de una buena negociación, en donde las partes se encuentren conformes con los resultados de la negociación y que serán formalizados mediante los instrumentos jurídicos que señalan los artículos 100 y 101 de la Ley de Hidrocarburos.

Ya analizamos Constitucionalmente el concepto de propiedad de la tierra al mencionar que le corresponde originalmente a la Nación $\mathrm{y}$ que por derecho la hace pasar a una persona (llamada propietario) (De Pina, 1995, p. 423) y así constituirse la propiedad, además que el suelo es considerado como un bien inmueble y que puede tener adhesiones fijas de bienes muebles que se convierten en parte integral del terreno, ahora definiremos a la propiedad desde cada una de las facultades jurídicas que la integran. La propiedad es el derecho de ejercer el poder directo e inmediato sobre un objeto o bien, por el que se le atribuye a su titular la capacidad de disponer del mismo, sin más limitaciones que las que imponga la ley, es el derecho real que implica el ejercicio de las facultades jurídicas más amplias que el ordenamiento jurídico concede sobre un bien (Morán, 2002) ${ }^{3}$.

Ahora bien, el objeto del derecho de propiedad pleno ${ }^{4}$ está conformado por bienes con posibilidad de apropiación y se requiere de tres condiciones: que sea útil, ya que ha de tener un fin; que el bien pueda ser cuantificado, y que sea considerado para ocupación, según Rodríguez Piñeres, al ejercer el derecho de propiedad pleno debe de tomarse en cuenta tres facultades principales: uso (ius utendi), goce (ius fruendi) y

3. El artículo 544 del Código Civil francés establece que "La propiedad es el derecho de gozar y disponer de una cosa de la manera más absoluta, siempre que no se haga de ella un uso prohibido por las leyes o por los reglamentos".

4. Al ejercer el derecho de propiedad el propietario debe de tener reunidas las tres facultades. 
disfrute (ius abutendi), mismas que describimos a continuación (1973, p. 70).

El (ius utendi) es el derecho de uso sobre el bien. El propietario tiene el derecho a usar o servirse de la cosa para sus propósitos e intereses de acuerdo con el derecho, siempre y cuando esos propósitos o intereses no violenten los preceptos legales ya establecidos o causen lesiones a los derechos de otros propietarios (p. 70).

El (ius fruendi) es el derecho de goce sobre un bien. El propietario tiene el derecho de aprovechar y disponer de los frutos o productos que son generados por el bien. Se desprende de lo anterior que el propietario de una cosa es por consiguiente dueño de todo aquello que la cosa produzca, con o sin su intervención. Y los frutos o ganancias podrán ser tanto naturales como civiles (p. 70).

El (ius abutendi) es el derecho de disposición sobre un bien. El propietario ejerce el poder que le faculta de tener el dominio sobre el bien, puede disponer como a su voluntad o intereses convenga, incluyendo dañarla o destruirla, salvo que esto tenga una repercusión en contra de terceros. Son dos tipos de disposiciones que se desprenden de este derecho: por un lado, el derecho a disponerlo materialmente; y el otro, el derecho a disponerlo en forma legal (p. 70).

En ese sentido, para que un propietario pueda hacer uso del derecho de propiedad de un bien, dependerá de la facultad o facultades de disposición ejercidas, para el uso o fin legal que quiera disponer del bien y cuando esta o estas disposiciones se comprometan con otra persona, dará como resultado las figuras jurídicas con las que se llegue a un acuerdo, y esto se traducirá en el alcance o restricción sobre la disposición del bien por parte del propietario y de otra persona de hacer uso o disposición del bien.

\subsection{Marco institucional y jurídico en} materia de uso y ocupación superficial

Entre las diversas transformaciones suscitadas de la multicitada reforma, el marco institucional en materia energética ha sufrido variaciones. Entre los principales actores, podemos citar a la Secretaría de Energía (SENER), la Secretaría de Desarrollo Agrario, Territorial y Urbano (SEDATU), la Comisión Nacional de Hidrocarburos
(CNH) y el Instituto de Administración y Avalúos de Bienes Nacionales (INDAABIN).

A continuación se presenta un esquema sobre la normativa aplicable en materia de uso y ocupación superficial, en la que se exponen cronológicamente, de acuerdo con la ejecución de la reforma, tanto las instituciones como los instrumentos normativos involucrados.

En primera instancia, la Secretaría de Energía $(\mathrm{SENER})^{5}$ conduce la política energética del país, dentro del marco constitucional vigente, para garantizar el suministro competitivo, suficiente, de alta calidad, económicamente viable y ambientalmente sustentable de energéticos que requiere el desarrollo de la vida nacional.

La Secretaría de Desarrollo Agrario, Territorial y Urbano (SEDATU) es una Secretaría de Estado encargada de la realización de planes, programas $\mathrm{y}$ proyectos vinculados al uso del territorio nacional para el asentamiento de comunidades rurales o urbanas, extensiones de tierra y agua. Entre sus atribuciones destaca la aplicación de la legislación agraria derivada del artículo 27 de la Constitución ${ }^{6}$.

El Instituto de Administración de Avalúos de Bienes Nacionales (INDAABIN) es un organismo público desconcentrado de la Secretaría de Hacienda y Crédito Público, cuyo objetivo es administrar y valuar el patrimonio inmobiliario federal y paraestatal, así como procurar una Administración Pública que cuente con los inmuebles adecuados para el pleno ejercicio administrativo ${ }^{7}$.

A continuación, se presenta un esquema cronológico en el que se exponen los principales instrumentos normativos; así como, las Secretarías de Estado, órganos reguladores y otras instituciones involucradas en uso y ocupación superficial $^{8}$.

5. Gobierno de México, Instituciones. Gobierno de México, Secretaría de Energía. Actividad, conjunto de datos.

6. Artículo 41 de la Ley de Orgánica de la Administración Pública Federal. 29 de diciembre de 1976. Consultado el 1 de enero de 2019. Edición actualizada con las reformas vigentes publicadas al 30 de noviembre de 2018.

7. Gobierno de México, Instituciones. Gobierno de México, Instituto de Administración y Avalúos de Bienes Nacionales.

8. Dado lo extenso de los documentos, se proporcionan los enlaces para su consulta en el apartado de Bibliografía. 


\section{Normativa aplicable en materia de uso y ocupación superficial}

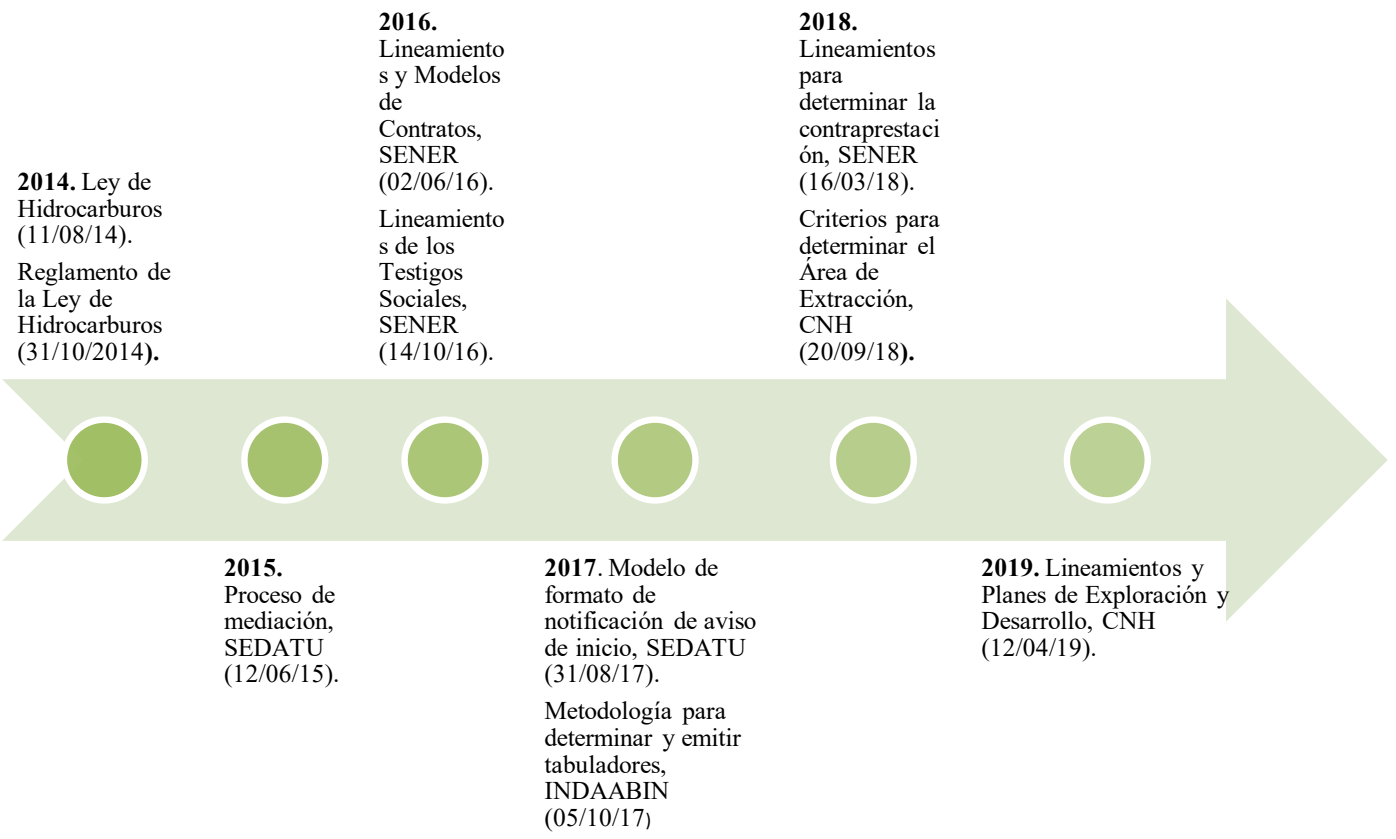

González Rodríguez, Carla Gabriela, Ocupación superficial en actividades de exploración y extracción de hidrocarburos, Comisión Nacional de Hidrocarburos, (s/f), Disponible en https://www.gob.mx/cms/uploads/attachment/file/468361/ PI_tica_11._Ocupaci_n_Superficial_en_Actividades_de_E_E_de_Hidrocarburos.pdf

Merece una descripción especial La Ley de Hidrocarburos, para efectos de este tema en particular, debido a que es el instrumento jurídico elemental para los procesos de extracción y producción de los hidrocarburos, que señala las condiciones que regularán el uso y la ocupación superficial de los terrenos susceptibles para la exploración, extracción y producción de la industria petrolera.

Artículo 36. Los Asignatarios y Contratistas deberán contar con autorización de la Comisión Nacional de Hidrocarburos, conforme a la regulación y los lineamientos que para tal efecto emita la citada Comisión, para llevar a cabo la perforación de los pozos en los casos siguientes:

\section{Pozos exploratorios;}

II. Pozos en aguas profundas y ultra profundas, $y$
III. Pozos tipo que se utilicen como modelos de diseño.

La autorización a que se refiere este artículo se ajustará a los plazos establecidos conforme a la regulación que para tal efecto emita la Comisión Nacional de Hidrocarburos. En caso de no emitirse una respuesta a la solicitud por parte de la Comisión Nacional de Hidrocarburos, dentro del plazo establecido en dicha regulación, ésta se entenderá en sentido favorable.

La Ley de Hidrocarburos en el artículo 101, señala las figuras jurídicas mediante las cuales se llevaran a cabo las negociaciones y acuerdos a que se refiere el artículo 100 de esta misma Ley y que hace referencia a las contraprestaciones, términos y condiciones para el uso, goce o afectación de los terrenos, bienes o derechos necesarios para realizar las actividades de exploración y extracción de hidrocarburos, que serán negociados entre los propietarios o titulares de los terrenos, bienes o derechos, con los asignatarios o contratistas, 
debiendo realizarse de manera transparente y sujetándose a las bases siguientes: la forma o modalidad de uso, goce, afectación, en su caso, adquisición que se pacte esta deberá de ser idónea para el desarrollo del proyecto en cuestión', según sus características, podrán ser empleadas las figuras de arrendamiento, servidumbre voluntaria, ocupación superficial, ocupación temporal, compraventa, permuta y cualquier otra que no contravengan a la ley (Art. 101, fracción V).

La figura arcaica, utilizada anteriormente y empleada por años, llamada Contrato de Ocupación Superficial, pero ahora plasmada en la Ley de Hidrocarburos, es una figura utilizada con fundamento, en la ahora abrogada Ley Reglamentaria del Artículo 27 Constitucional en el Ramo del Petróleo, pero que en toda esta Ley no existe tal figura, ya que dicho precepto hace referencia a una ocupación provisional y no a una ocupación superficial y, que apegado a derecho la forma en que pudiera ser convenida esta modalidad contractual, debería de ser bajo otro seudónimo de contrato, para no dar más rodeos, esta figura carece de sustento legal, ya que estaríamos confundiendo entre una renta, compra-venta o servidumbre de paso.

No muy lejana a la anterior definición y para mayor confusión, un contrato temporal es una figura jurídica que se encuentra mencionada y reglamentada en la Ley de Expropiación, por lo que estaríamos hablando de una declaración unilateral de voluntad de imposición por parte del Estado, sobre el bien del propietario de imponer una limitación, lo cual resulta contradictorio a una negociación entre las partes como lo señalan los artículos 100 y 101 de la Ley de Hidrocarburos.

En este nuevo esquema para el uso y ocupación superficial se contempla un abanico de opciones legales que son enunciativas, mas no limitativas,

9. Dependiendo las características del proyecto y las negociaciones serán las figuras jurídicas para el uso, goce y afectación las cuales se describen a continuación:

Arrendamiento. Contrato en virtud del cual una parte cede a la otra el uso y disfrute de una cosa o derecho, mediante un precio cierto, que recibe la denominación de renta o alquiler.

Servidumbre voluntaria. Primero, ¿qué es servidumbre? Gravamen real impuesto sobre un inmueble en beneficio de otro perteneciente a distinto dueño (art.1057 a 1134 del Código Civil para el Distrito Federal). Y servidumbre voluntaria es el uso y la extensión de las servidumbres establecidas por la voluntad del propietario, se arreglarán por los términos del título en que tengan su origen. al dejar abierta la opción de cualquier otra que no contravenga ${ }^{10}$ la ley (Mabarak, 1995, p. 53) y por las cuales podrán optar las partes para garantizar los trabajos y actividades que se llevaran a cabo en los terrenos de los propietarios de la tierra, con las limitantes que en los mismos acuerdos convengan las partes.

En lo que concierne al Reglamento de Hidrocarburos, es importante describir los artículos 6 y 7 del Título Segundo, que trata del Reconocimiento y Exploración Superficial y Extracción de Hidrocarburos, los cuales señalan:

Artículo 6.- Las actividades de Reconocimiento y Exploración Superficial requieren de autorización de la Comisión de conformidad con la regulación que ésta emita para tal efecto. No requiere de autorización para realizar actividades de Reconocimiento y Exploración Superficial lo previsto en el último párrafo del artículo 37 de la Ley.

La empresa productiva del Estado o la persona que pretenda obtener la autorización a que se refiere este artículo, deberá presentar a la Comisión una solicitud que contenga lo siguiente:

I. La descripción general del proyecto, la cual deberá incluir el objeto y alcance;

II. Las áreas en las que se pretende realizar el levantamiento de datos de campo;

III. La descripción del plan de adquisición de datos a realizar;

IV. La descripción de la tecnología que se utilizará para la adquisición de datos;

V. El análisis de riesgos técnicos para el caso de adquisición de datos en campo;

VI. El programa de entrega de datos de campo, procesados, reprocesados y sus interpretaciones, según sea el caso;

VII. La demás información que solicite la Comisión con base en la regulación que emita.

10. Se refiere a que no sean carentes de sustento legal los convenios entre las partes al incurrir en alguna irregularidad fuera de la legalidad del derecho civil o mercantil. 
Artículo 7.- El aviso a que se refiere el último párrafo del artículo 37 de la Ley se sujetará a la regulación que al efecto emita la Comisión y deberá incluir lo previsto en las fracciones III a VII del artículo anterior. Esto último será aplicable solamente tratándose de actividades que en su totalidad se realicen dentro del Área de Asignación o el Área Contractual.

1.2 Posibles repercusiones económicas, sociales y administrativas derivadas del ejercicio de la imposición del interés público sobre la propiedad para el aprovechamiento de los recursos energéticos

\subsubsection{Posibles repercusiones negativas}

La primera repercusión de esta naturaleza, sería la disminución de un área proporcional de los terrenos de los propietarios, ya que al realizarse los trabajos para la exploración y extracción de los hidrocarburos, se vería comprometida y mermada la cantidad de terreno con el que cuenta la propiedad, lo que generaría una disminución en el derecho de uso del propietario.

Otra situación, sería la interrupción de los ciclos de siembra y pastas para ganado, ya que, por resultado lógico el bien inmueble que se afecta con las actividades de extracción de los hidrocarburos estarán imposibilitados para continuar generando producción agrícola o forestal que sirve de comercialización de productos o alimento para los animales de crianza. Por lo tanto, al ser mermada la producción agrícola y ganadera como cualquier otra que se lleve a cabo en el campo, significará una disminución de los ingresos de los propietarios o poseedores de la tierra.

Existen daños colaterales en las operaciones de los equipos que son trasladados por las compañías que realizaran los trabajos, así como del personal que realiza las actividades durante la exploración y extracción de hidrocarburos que transitan por los caminos vecinales cuando no son pavimentados, lo que hace que la vegetación se vea afectada por partículas del camino (polvo) lo que provocará que la vegetación se seque y escasee el alimento para los animales de crianza.

La perturbación social por la invasión a los usos y costumbres regionales, de las comunidades rurales y propietarios particulares. La invasión a la propiedad es preponderantemente inevitable como también lo es la interacción que se tenga por parte del personal de las compañías perforadoras o proveedoras con los residentes pobladores, lo que trae la molestia de las comunidades que habitan los lugares donde los trabajos son desarrollados, provocando descontento e inconformidades, algunas de las causas que también se presentan en estos problemas con las comunidades residentes, son la contaminación por basura, daños a caminos y linderos de propiedad, percances y atropellamiento de animales de crianza semovientes o en casos severos con los mismos pobladores, al no respetar los límites de velocidad establecidos para las brechas o los caminos vecinales que conducen tanto a las comunidades como a las áreas de trabajo.

\subsubsection{Posibles repercusiones positivas}

Con la necesidad de transitar por las vías de comunicación de las comunidades en donde se desarrollen los trabajos para la extracción de hidrocarburos, se optará por la construcción de caminos con mejores materiales de revestimiento o en su caso, hasta la pavimentación lo que da el beneficio de contar con mejores vías de comunicación para los pobladores y así poder sacar de sus lugares de origen los artículos que en un momento dado podrían comercializar y tener un ingreso.

Otro punto, sería el desarrollo de negocios locales en el lugar donde se desarrollen las actividades de extracción de recursos energéticos, ya que con el incremento constante del personal de las compañías encargadas de desarrollar las actividades petroleras, se propiciarán necesidades y oportunidades a los negocios locales, los cuales se verán beneficiados, como por ejemplo, los de hospedaje, restaurantes e insumos en general para satisfacer las necesidades de las compañías.

Uno de los beneficios directos que tiene el desarrollo de las actividades petroleras y trabajos periféricos, es la creación de fuentes de empleo, ya que son satisfechos con las mismas personas que se encuentran residiendo en el lugar donde se desarrollan estos trabajos, lo que beneficia a las personas oriundas con capacitación y adiestramiento.

Todo lo descrito en materia de repercusiones negativas es una realidad a la que nos enfrentamos actualmente, sin embargo, se espera que se corrijan en un futuro mediato y, que los aspectos positivos que existen se acrecienten. 
Además de las situaciones negativas ya descritas, algunas posibles complicaciones que se pudieran presentar en las negociaciones y acuerdos para el uso y ocupación superficial, tanto en la propiedad privada como rural, son:

a) Que no se lleve a cabo el acuerdo de negociación entre las partes por cuestiones, ya sean de inconformidad en el avalúo Porción o en la forma del terreno que se afectará.

b) Que se pudieran presentar en las negociaciones y acuerdos para el uso y ocupación superficial, tanto en la propiedad privada como rural.

c) La sobrecarga de trabajo y falta de capacitación en la materia especializada por parte de los Juzgados de Distritos o Tribunales Agrarios para ejercer el procedimiento de impartición de la justicia por daños causados en la explotación de recursos energéticos, vayan retrasando las negociaciones $y$,

d) Las carencias de documentos que justifican la propiedad por parte del tenedor de la tierra.

e) Las presiones sociales que bloqueen en total o parcialmente los procesos de negociación.

También se observa que comúnmente se presentan estas situaciones en núcleos de población rural por falta de un comisariado ejidal que represente los intereses comunes del ejido y esto es porque dentro de la misma comunidad se generan riñas entre los mismos pobladores, lo que ha provocado que durante años o nunca haya existido una representación, ya que es imposible llegar a una convocatoria para una elección del órgano representante del ejido.

\section{VÍAS Y PROCESOS NORMATIVOS EN MATERIA DE USO Y OCUPACIÓN SUPERFICIAL EN HIDROCARBUROS}

Ante el escenario planteado por la reforma energética, se vislumbra que la ejecución de proyectos energéticos podría suscitar controversias entre los actores involucrados (comunidades y empresas, principalmente), por lo cual se han establecido vías y procesos que resuelvan los posibles conflictos.
Haciendo énfasis en la mediación, la SEDATU impulsa la formación del mediador energético, cuya figura surge tras su incorporación a la Ley de Hidrocarburos y de la Industria Eléctrica, que forma parte de la reforma energética. La nueva legislación faculta a la SEDATU a intervenir, a petición de alguna de las partes, en los procesos de negociación y mediación para el uso y utilización de la tierra para el desarrollo de los proyectos energéticos (2016), y esto es así, porque la gente sabe lo que vale su tierra, y es conveniente que tenga un pago justo por ello o una renta justa por ella.

La SEDATU establece los lineamientos para que pueda llevarse a cabo una mediación:

1. La razón o denominación social del solicitante, debiendo acompañar original o copia certificada del acta constitutiva y original, o copia certificada de la escritura pública donde conste poder o mandato;

2. El domicilio del solicitante;

3. La descripción del proyecto energético y el carácter con el que desarrollará el mismo, debiendo acreditar dicho carácter;

4. La ubicación del proyecto energético que se pretende desarrollar, incluyendo coordenadas UTM, vías de acceso, referencias de campo y acompañada de un plano de ubicación;

5. el nombre del propietario o titular del terreno, bien o derecho de que se trate al que se notificó el interés de adquirir, usar, gozar o afectar los mismos;

6. la descripción detallada de las negociaciones (si la hay) llevadas a cabo, incluyendo propuesta de contraprestación hecha, razones de la negativa, identificación de actores internos y/o externos y todas aquellas personas físicas o morales que hayan influido en la toma de decisiones o cualquier tipo de acuerdo que haya surgido entre las partes, entre otros: lugar para llevar a cabo las reuniones, plazos, lengua o idioma;

7. Los avalúos en términos del artículo 104 de la Ley de Hidrocarburos realizados 
unilateralmente por alguna de las partes en los términos señalados;

8. $\quad$ La copia de la notificación realizada a la Secretaría del inicio de las negociaciones, a que se refiere el artículo 101 fracción IV de la Ley;

9. El estudio de Impacto Social; y

10. Cualquiera otra información que a criterio del solicitante resulte relevante para la Mediación (Diario Oficial de la Federación, 2015).

Este es uno de los principales lineamientos, pero solo se refiere a los requisitos que deben cumplir los participantes que desean llevar a cabo una mediación, una conciliación. Las mesas de diálogo que seguramente se hayan establecido, sus implicaciones jurídicas y resultado no están, desafortunadamente, a la vista en los portales correspondientes. Por otra parte, tampoco se tiene el número de asuntos que se hayan tramitado bajo esta figura.

Si bien es cierto lo antes descrito, también lo es que una vez que esta figura se ponga en marcha en la actual administración rendirá frutos muy importantes para las partes involucradas, pero principalmente para los débiles de la relación, los propietarios o poseedores de la tierra.

\section{LA SOCIEDAD Y LA SUSTENTABILIDAD EN LA PRODUCCIÓN DE ENERGÉTICOS}

Las leyes secundarias derivadas de la reforma energética han establecido una serie de cambios en la legislación, con la finalidad de atraer inversiones para modernizar el sector energético, con el objetivo de impulsar el crecimiento económico de la nación y hacerlo sustentable (Reformas, 2015).

Por ello, se estableció en el artículo 25, párrafo sexto que:

Bajo criterios de equidad social, productividad y sustentabilidad se apoyará e impulsará a las empresas de los sectores social y privado de la economía, sujetándolos a las modalidades que dicte el interés público y al uso, en beneficio general, de los recursos productivos, cuidando su conservación y el medio ambiente.

Esto es un referente que da fundamento y motivación legal con un nivel de importancia por igual, entre los temas económicos, sociales, ambientales, a los que metafóricamente podríamos poner en una balanza de tres platos o niveles y el resultado serían proyectos sustentables, ya que cada uno de los temas tiene ahora un nivel por igual de importancia, lo que se traduce como sustentabilidad.

Actualmente, el concepto de sustentabilidad (Centro del Cambio y Sustentabilidad, 2013) ha retomado gran popularidad a nivel mundial. Como antecedente histórico, el concepto surgió en 1987, cuando la World Commission on Environment and Development de las Naciones Unidas publicó el Informe Brundtland "Our common future" (Nuestro futuro común) (Gómez, G. 2014), el cual se enfoca en la idea del desarrollo sustentable o sostenible. Sin embargo, fue hasta 1992 en Río de Janeiro, durante la Conferencia de las Naciones Unidas sobre el medio ambiente y desarrollo, que 180 Jefes de Estado adoptaron el concepto mediante el acuerdo de 27 principios relacionados con la Sustentabilidad, materializándose en un programa mundial conocido como Agenda 21.

En el Informe Brundtland, se estableció que: "El desarrollo sustentable hace referencia a la capacidad que haya desarrollado el sistema humano para satisfacer las necesidades de las generaciones actuales sin comprometer los recursos y oportunidades para el crecimiento y desarrollo de las generaciones futuras" (Gómez de S., s. f.).

Por otra parte, en el estudio "El concepto moderno de la sustentabilidad"11, efectuado por Arturo M. Calvente (2007) de la Universidad Abierta Interamericana, se menciona una definición sueca más integral, al definir una sociedad sustentable como aquella en la cual: "el desarrollo económico, el bienestar social y la integración están unidos con un medio ambiente de calidad. Esta sociedad tiene la capacidad de satisfacer sus necesidades actuales sin perjudicar la habilidad de que las generaciones futuras puedan satisfacer las suyas".

Este nuevo criterio de sustentabilidad, ha sido tomado en consideración en las leyes

11. En el mismo estudio, se plantea una definición más desde el punto de vista económico: "sustentabilidad es la habilidad de lograr una prosperidad económica sostenida en el tiempo protegiendo al mismo tiempo los sistemas naturales del planeta y previendo una alta calidad de vida para las personas". 
secundarias, por lo que tanto las cuestiones administrativas, laborales, económicas, ambientales y sociales contempladas en estas leyes dependen unas de otras por estar ligadas al principio de sustentabilidad, ahora con un rango constitucional.

Por ello, la Ley de Hidrocarburos señala requisitos y formalidades que deben de cumplirse (artículo 100 de la Ley de Hidrocarburos) sobre los aspectos sociales y medio ambiente, uno de los temas ya conocidos era el impacto ambiental, pero el que se une a la ecuación es el impacto social, mismo que tiene un apartado específico al describir que los interesados en obtener un permiso o una autorización para desarrollar proyectos en materia de Hidrocarburos, tanto los Asignatarios y Contratistas, deberán presentar a la Secretaría de Energía una evaluación de impacto social que deberá contener la identificación, caracterización, predicción y valoración de los impactos sociales que podrían derivarse de sus actividades, así como las medidas de mitigación y los planes de gestión social correspondientes, en los términos que señale el Reglamento de la Ley (Art. 121).

Las evaluaciones de impacto permiten medir el uso de metodologías rigurosas, los efectos que un programa puede tener sobre su población beneficiaria y conocer si dichos efectos son en realidad atribuibles a su intervención. El principal reto de una evaluación de impacto es determinar qué habría pasado con los beneficiarios si el programa no hubiera existido.

\subsection{Impacto social}

La sociedad tiene un rol fundamental en el tema de la sustentabilidad en la producción de energéticos, es por ello que se debe aludir a varios términos importantes como el del impacto social.

La utilización del término "impacto social" se ha ampliado y ha sido objeto de múltiples definiciones en la literatura, referida a los problemas sociales, entre las que destacan:

El impacto se refiere a los efectos que la intervención planeada tiene sobre la comunidad en general. Asimismo, puede verse en la forma cómo se realiza el proceso o las prácticas utilizadas y que dependen, en gran medida, de quienes lo ejecutan.
La definición realizada por la Organización de las Naciones Unidas en 1984, expresa que la evaluación constituye el proceso en que se determina, de manera sistemática y objetiva, la pertinencia, eficiencia, eficacia e impacto de las actividades en relación con los objetivos planteados para la realización de éstas (Urrutia en Libera, 2007).

Las evaluaciones de impacto permiten medir por medio del uso de metodologías rigurosas, los efectos que un programa puede tener sobre su población beneficiaria y conocer si dichos efectos son en realidad atribuibles a su intervención. El principal reto de una evaluación de impacto es determinar qué habría pasado con los beneficiarios si el programa no hubiera existido.

Una vez descritos la sustentabilidad y el impacto social se hace referencia mediante la siguiente gráfica, de cómo pueden armonizar en forma equilibrada la equidad (social, económica y ambiental), el crecimiento económico y la sustentabilidad ambiental, a lo que tenemos como resultado un desarrollo sustentable (Constitución política, Art. 25) que en esta imagen es al centro del triángulo (Barrios, 2016).

Gráfica 1. El Triángulo de Peter Nijkamp (Zarta, 2017, p. 415)

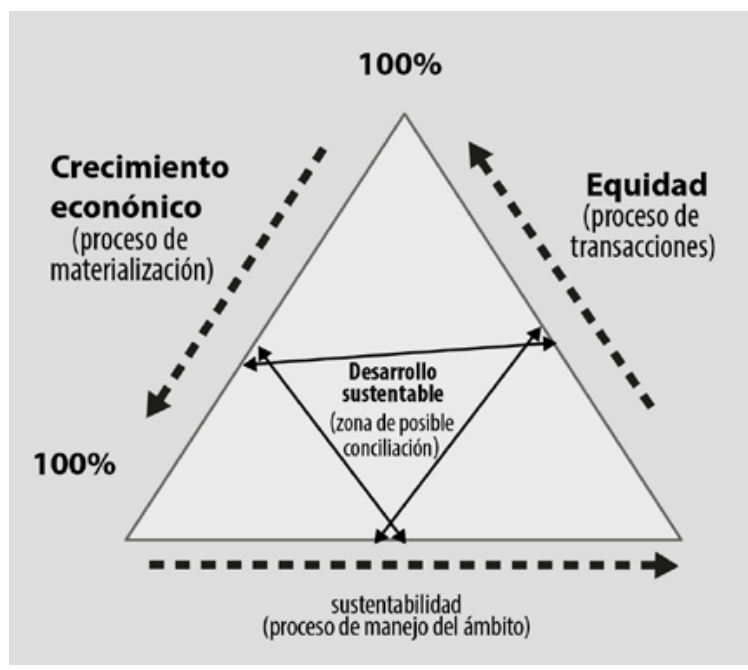

Por lo tanto, una producción con sustentabilidad, socialmente equitativa $\mathrm{y}$ responsable basada en un crecimiento 
económico, da como resultado un desarrollo sustentable. Esto es que mediante la evolución tecnológica la industria incrementa su potencial de producción, para satisfacer las necesidades humanas con productos y fuentes de empleos justos y socialmente equitativos, respetando y haciendo buen uso del medio ambiente y sus recursos naturales, lo que da como resultado un crecimiento económico responsable y socialmente equitativo con una producción con sustentabilidad.

\section{COMENTARIOS FINALES}

A raíz de la aparición de la legislación secundaria de la reforma energética, publicada en el Diario Oficial de la Federación en agosto de 2014, el uso y ocupación superficial de las tierras para el desarrollo de los trabajos de exploración, extracción y producción de hidrocarburos, ahora posee un apartado detallado en el cual, la figura jurídica de la tierra de los propietarios o poseedores tendrán las facultades de ejercer lo que, conforme a derecho, se conceda y convenga, para la negociación y así lograr el mejor aprovechamiento de las tierras, sin dejar de lado la facultad de la nación de ejercer el derecho de propiedad original que tiene sobre la tierra conferida para el aprovechamiento de los recursos naturales para el bien común de la población, de acuerdo a la Constitución Política de los Estados Unidos Mexicanos.

Lo anterior, representa un avance transcendental, ya que por mucho tiempo, la utilización de una figura jurídica carente de fundamento legal, así como la falta de equidad entre las partes contratantes, cláusulas inamovibles, la imposición de un avalúo al bien inmueble utilizando un organismo propio del Estado, fueron constantes problemáticas en las prácticas de uso y ocupación superficial en las instalaciones donde se realizaban proyectos energéticos.

En el nuevo planteamiento derivado de la reforma energética, se enumera una serie de mecanismos jurídicos con intervención de organismos privados y de gobierno para la observación a las bases que deberán cumplirse durante el proceso, para formalizar el uso y ocupación de tierras destinadas al aprovechamiento de los energéticos. Este enorme salto, entre un proceso y otro, no es más que la respuesta a que ya no solo las empresas del
Estado serán las únicas que podrán llevar a cabo las actividades que eran antes exclusivas; hoy, las contratistas (empresas particulares) podrán también llevar a cabo los trabajos en las áreas estratégicas antes reservadas solamente a las paraestatales, ahora EPE (Empresas Productivas del Estado), en lo que las condiciones se igualan al hacer una negociación con los propietarios de los inmuebles.

La relación entre contratistas y propietarios que se ha llevado en los últimos años después de 2013-2014, vislumbra una realidad social equitativa, legal y sustentable. Si enfocamos esta descripción de una manera optimista. Sin embargo, los cambios sociales, políticos y económicos que trae aparejado un cambio del Poder Ejecutivo sexenal ha detenido, administrativamente hablando, los posibles acuerdos a que se estaban llegando los participantes a invitación de la SEDATU.

A agosto de 2019, no se puede decir, si ahora sí habrá una importante ayuda a los más necesitados de esta relación, que sin dudarlo, son los propietarios, poseedores de la tierra $y$, sí también habrá mecanismos inteligentes que coadyuven a este propósito.

Aunado a lo anterior, otras problemáticas se han detectado a la fecha, entre las cuales se encuentran:

- Los pasivos sociales y negativos a negociar

- La corta vigencia de acuerdos y largos procesos para llegar a cosa juzgada

- La ausencia de titular de los derechos de la tierra (falta de registros, intestados)

- El número de propietarios y consideración del área de extracción comercial

- La definición para compartir la contraprestación

- Las altas expectativas de contraprestación (Zarta, 2017, p. 415).

Si bien la Ley de Hidrocarburos y su Reglamento están para proporcionar un marco jurídico garantista de los derechos de los 
propietarios, también uno de los objetivos es brindar certeza jurídica al contratista, por lo que se requiere hacer difusión de los beneficios del desarrollo de los proyectos energéticos para los propietarios de los derechos de la tierra. Para ello, es fundamental la creación de una base de datos interinstitucional en materia de ocupación superficial, para dar seguimiento al proceso, desde el aviso de negociación hasta su conclusión.

Es necesario también, el fortalecimiento de atribuciones de las distintas dependencias y organismos para atender temas relacionados con ocupación superficial mediante la organización de talleres técnicos de SENER, SEDATU, INDAABIN y CNH para homologar los criterios del proceso y atender problemáticas específicas de los operadores petroleros.

Se está en un período de observación de hechos sociales que repercuten irremediablemente a lo jurídico, y aún no se puede dar una información final. Solo se reitera que ojalá el gobierno actual utilice de la mejor manera posible los instrumentos jurídicos que ya tiene para que se haga realidad una verdadera justicia social en México.

\section{BIBLIOGRAFÍA}

- Barrios Vera, J. G. (s/f) Sostenibilidad económica y social como prioridad para la sustentabilidad ambiental. Gestiopolis., Disponible en http://www.gestiopolis. com/sostenibilidad-economica-socialprioridad-sustentabilidad-ambiental/

- Instituto de Investigaciones Jurídicas, UNAM. (s/f). Biblioteca Jurídica Virtual. Disponible en http://biblio.juridicas.unam.mx/

- Calvente, A. M. (2007). El concepto moderno de sustentabilidad, UAIS Sustentabilidad. Disponible en: https://n9.cl/kjt92x.

- Cámara de diputados. Servicios de Investigación, Marco Jurídico Conceptual. Disponible en http://www.diputados. gob.mx/bibliot/publica/inveyana/polint/ cua1/mj-1.htm

- Centro del cambio y sustentabilidad, A.C. (2013). ¿Qué es sustentabilidad?. Disponible en http://ccgss.org/sustentabilidad/

- Clúster de Energía Coahuila, A.C. (2019). Impacto social y ocupación superficial. Disponible en https://clusterenergia.org/ isos/

- Código Civil Federal. (2013). Diario Oficial de la Federación.

- Consejo Nacional de Evaluación de Política de Desarrollo Social. Disponible en: https:// n9.cl/sy $2 \mathrm{~kg}$.

- Constitución Política de los Estados Unidos
Mexicanos. (2015). Diario Oficial de la Federación.

- De Pina V., R. (2015). Diccionario de Derecho, México: Porrúa.

- Díaz L., R. M. (2014). El Ombudsman de la Salud en México. México: Instituto de Investigaciones Jurídicas/UNAM.

- Instituto de Investigaciones Jurídicas, UNAM (1996). Diccionario jurídico mexicano. México: Porrúa.

- Gobierno de México. (2019) Instituto de Administración y Avalúos de Bienes Nacionales. Disponible en https://datos. gob.mx/busca/organization/about/ indaabin

- Gobierno de México (2019). Secretaría de Energía. Actividad. Disponible en https:// datos.gob.mx/busca/organization/about/ sener

- Gómez de S. B., R., (s. f.). Del desarrollo sostenible según Brundtland a la sostenibilidad como biomimesis. Bilbao: Universidad del país Vasco Disponible en https://www.upv.es/contenidos/ CAMUNISO/info/U0686956.pdf

- Gómez G., C. (2019). El desarrollo sostenible: conceptos básicos, alcance y criterios para su evaluación. Nueva York: UNESCO.

- González R., C. G. (s. f.) Ocupación superficial en actividades de exploración y extracción 
de hidrocarburos. Comisión Nacional de Hidrocarburos, Disponible en https:// www.gob.mx/cms/uploads/attachment/ file/468361/Pl_tica_11._Ocupaci_n_ Superficial_en_Actividades_de_E_E_de_ Hidrocarburos.pdf

- Indian and northern affairs Canada. (2003). Northern Land Use Guidelines. Overview. Minister of Public Works and Government Services Canada. Disponible en: https:// n9.cl/85enx.

- Ley de hidrocarburos. Diario Oficial de la Federación. 13 de agosto de 2014.

- Ley de la Agencia Nacional de Seguridad Industrial y de Protección al Medio Ambiente del sector hidrocarburos. (2014). Disponible en: http://www.diputados.gob. $\mathrm{mx} /$ LeyesBiblio/pdf/LANSI_110814.pdf

- Ley de Orgánica de la Administración Pública Federal. (29 de diciembre de 1976). Actualizada con las reformas vigentes publicadas, al 30 de noviembre de 2018.

- Mabarak C., D. (1995). Derecho Financiero Público. México: Mc Graw Hill.

- Morán M., R., (2002). Los derechos sobre las cosas (I). El derecho de propiedad y derecho de posesión. En: Historia del Derecho Privado, Penal y Procesal. Tomo I. Parte teórica. México: Editorial Universitas.

- Reglamento de la Ley de Hidrocarburos. (31 de octubre de 2014). Diario Oficial de la Federación.

- Rodríguez P., E. (1973). Derecho usual. Bogotá: Temis.

- Rojina V., R., (1994). Compendio de Derecho Civil, Bienes Derechos Reales y Sucesiones. México: Porrúa.

- Ruiz M., M. (1990). Derecho Agrario. Universidad Nacional Autónoma de México, Instituto de Investigaciones Jurídicas. Disponible en http://biblio.juridicas.unam. $\mathrm{mx} /$ libros/libro.htm?l=281

- Secretaría de Desarrollo Agrario, Territorial y Urbano. (2016). Impulsa SEDATU formación de mediadores energéticos con visión pública: Rosario Robles. Disponible en. https://n9.cl/e0pf.

- Secretaría de Gobernación, (12 de abril de 2019). Lineamientos de Planes de Desarrollo, CNH, Diario Oficial de la Federación, Disponible en https://n9.cl/t9jh.

- Secretaría de Gobernación, (9 de marzo de 2018). Lineamientos y Modelos de Contratos, SENER, Diario Oficial de la Federación, Disponible en https://n9.cl/la2fe.

- Secretaría de Gobernación. (12 de marzo de 2018). Lineamientos para determinar la contraprestación, SENE, Diario Oficial de la Federación. Disponible en http://www.dof. gob.mx/nota_detalle.php?codigo $=5516472$ \&fecha $=16 / 03 / 2018$

- Secretaría de Gobernación (20 de septiembre de 2018), Criterios para determinar el Área de Extracción, CNH, Diario Oficial de la Federación. Disponible en https://n9.cl/gv1up.

- Secretaría de Gobernación. (5 de octubre de 2017). Metodología para determinar y emitir tabuladores, INDAABIN, Diario Oficial de la Federación. Disponible en https:// n9.cl/dcrqu.

- Secretaría de Gobernación. (31 de agosto de 2017). Modelo de Formato de Notificación de Aviso de Inicio, SEDATU, Diario Oficial de la Federación. Disponible en https://n9.cl/ d814m.

- Secretaría de Gobernación. (14 de octubre de 2016). Lineamientos de los Testigos Sociales, SENER, Diario Oficial de la Federación. Disponible en https://n9.cl/ $\operatorname{lm} 0 \mathrm{u} 5$

- Secretaría de Gobernación, (12 de junio de 2015). Lineamientos que regulan el proceso de mediación sobre el uso y ocupación superficial en materia de hidrocarburos. Diario Oficial de la Federación, Disponible en https://n9.cl/gqrit

- Suprema Corte de Justicia de la Nación. (s.f.) Semanario Judicial de la Federación. Disponible en https://www.scjn.gob.mx/ 
Paginas/Inicio.aspx

- Urrutia B., L. (2007). El sistema de monitoreo y evaluación en contexto de los Proyectos APPL/PDL/UNIFEM. Observaciones no publicadas, En: Libera Bonilla, Blanca Esther. Impacto, impacto social y evaluación del impacto. ACIMED,
15(3). Disponible en https://n9.cl/rfabs, ISSN 1024-9435.

- Zarta Ávila, P. (2017). La sustentabilidad o sostenibilidad: un concepto poderoso para la humanidad, Tabula Rasa, (28): 409 423. http://doi.org/10.25058/20112742. n28.18 